\title{
Standard Model Prediction for Direct CP-violation in K-decays, and Long-Distance Contributions to Kaonic Amplitudes
}

\section{Kelly*}

Columbia University, 538 W 120th St., New York NY 10027

E-mail: ckelly@phys.columbia.edu

\begin{abstract}
Recent theoretical and computational advances have enabled the precision determination of multiparticle Standard Model amplitudes using lattice QCD. In particular the measure of direct CPviolation in kaon decays, $\varepsilon^{\prime}$, has now been computed with errors that are $40 \%$ of the experimental value; this quantity is highly sensitive to BSM sources of CP-violation and may help shed light on the origin of the matter-antimatter asymmetry in the Universe. We will discuss this calculation of $\varepsilon^{\prime}$ and our present and future efforts in improving our result. We will also briefly discuss our ongoing calculations of other long-distance contributions to kaonic amplitudes sensitive to new physics.
\end{abstract}

38th International Conference on High Energy Physics 3-10 August 2016

Chicago, USA

* Speaker. 


\section{Introduction}

Lattice QCD is a computational technique whereby low-energy hadronic interactions are directly simulated on supercomputers. It is the only known technique for studying low-energy QCD that is systematically improvable, meaning that all of the systematic error sources can be controlled and reduced with sufficient computational effort. With recent theoretical and algorithmic advances, and continued developments in computing power, lattice calculations have now reached a level of precision with which they can make a significant impact on the search for Beyond the Standard Model (BSM) physics. Of particular importance is the development of techniques for calculating matrix elements of multi-particle states, for which there are power-law finite-volume effects that must be eliminated. In this work we discuss the application of these techniques to several kaonic matrix elements involving multi-particle states that are known to be sensitive to BSM physics.

\section{2. $K \rightarrow \pi \pi$ and $\varepsilon^{\prime} / \varepsilon$}

The preponderance of matter over antimatter in the observable Universe can be explained by baryogenesis, a requirement for which is the breaking of the CP symmetry. While CP-violation $(\mathrm{CPV})$ is present in the Standard Model, its magnitude appears to be too small to account for the size of the matter/antimatter asymmetry, suggesting new physics awaits discovery. Direct CPV in K-meson decays is highly suppressed in the Standard Model and therefore offers a particularly sensitive probe for BSM sources of CPV.

Direct CPV in kaon decays was discovered in the late 1990s at Cern (NA31/NA48) and Fermilab $(\mathrm{KTeV})$ with the following result:

$$
\operatorname{Re}\left(\varepsilon^{\prime} / \varepsilon\right) \approx \frac{1}{6}\left(1-\left|\frac{\eta_{00}}{\eta_{ \pm}}\right|^{2}\right)=16.6(2.3) \times 10^{-4},
$$

where $\varepsilon^{\prime}$ and $\varepsilon$ are the measures of direct and indirect CPV, respectively, and $\eta_{i j}=A\left(K_{L} \rightarrow\right.$ $\left.\pi_{i} \pi_{j}\right) / A\left(K_{S} \rightarrow \pi_{i} \pi_{j}\right)$. However, until recently there has not been a reliable Standard Model prediction for this quantity that can be compared to experiment because the process receives large contributions from low-energy QCD interactions that are not amenable to perturbative calculations. The RBC \& UKQCD collaborations have now performed [1] the first complete, realistic calculation of $\varepsilon^{\prime}$ in the Standard Model, which we summarize below.

$\varepsilon^{\prime}$ can be computed on the lattice via

$$
\varepsilon^{\prime}=\frac{i e^{i\left(\delta_{2}-\delta_{0}\right)}}{\sqrt{2}} \frac{\operatorname{Re}\left(A_{2}\right)}{\operatorname{Re}\left(A_{0}\right)}\left(\frac{\operatorname{Im}\left(A_{2}\right)}{\operatorname{Re}\left(A_{2}\right)}-\frac{\operatorname{Im}\left(A_{0}\right)}{\operatorname{Re}\left(A_{0}\right)}\right) .
$$

where $A_{2}$ and $A_{0}$ are $K \rightarrow \pi \pi$ amplitudes where the final two-pion state has isospin $2(\Delta I=3 / 2)$ and $0(\Delta I=1 / 2)$, respectively. The quantities $\delta_{I}$ are the corresponding $\pi \pi$ scattering phase shifts.

At low energies the $K \rightarrow \pi \pi$ amplitudes are described very precisely by first-order $\Delta S=1$ weak effective theory:

$$
A_{I}=F \frac{G_{F}}{\sqrt{2}} V_{u s}^{*} V_{u d}\left[z_{i}(\mu)+\tau y_{i}(\mu)\right] Z_{i j}(\mu)\left\langle(\pi \pi)_{I}\left|Q_{j}(\mu)\right| K\right\rangle,
$$

where $F$ is the Lellouch-Lüscher factor [2] that represents the finite-volume correction to the decay amplitude, $z$ and $y$ are perturbative c-number Wilson coefficients, $\tau=-V_{t s}^{*} V_{t d} / V_{u d} V_{u s}^{*}, V_{i j}$ are CKM matrix elements, and $Q_{i}$ are a set of dimension-six four-quark operators. $Z_{i j}$ is the renormalization matrix relating the bare lattice operators to $\overline{\mathrm{MS}}$ operators normalized at the scale $\mu$, thereby matching the scheme used in the calculation of the Wilson coefficients. These are computed without resorting to the use of perturbative QCD at the hadronic scale by using an intermediate 'regularization-invariant momentum scheme' with symmetric kinematics (RI-SMOM) [3, 4] 
with which we can non-perturbatively run to a high energy scale where we can legitimately match to $\overline{\mathrm{MS}}$ using continuum perturbation theory.

The calculation of $A_{0}$ is particularly difficult due to the presence of so-called disconnected diagrams where the two-pion state decays into the vacuum and is recreated at a later time - such diagrams are typically very noisy. In order to obtain good statistical resolution we use so-called 'all-to-all' propagators [5], whereby an approximation to the quark propagator from any site to any other is obtained by exactly computing a subset of low eigenmodes and 'patching up' the highmode contribution using a stochastic technique. This allows us to tune the size and shape of the two-pion source to minimize the overlap of our operator with the vacuum, as well as to maximally translate the operators for optimal statistics.

A state is created on the lattice by the action on the vacuum of an operator with the desired quantum numbers. In practise this generates a linear combination of all allowed states with those quantum numbers. On a Euclidean lattice the more energetic states decay away exponentially in time, hence $K \rightarrow \pi \pi$ matrix elements involving the ground-states can be extracted simply by sufficiently separating the four-quark operator from the kaon and $\pi \pi$ operators in lattice time.

Unfortunately the ground-state of the $I=2 \pi \pi$ system comprises two pions at rest, and in the $I=0$ channel the same applies after the vacuum contribution is subtracted. This state has energy $2 \times m_{\pi} \sim 260 \mathrm{MeV}$, much smaller than the kaon mass of $\sim 500 \mathrm{MeV}$. The dominant contribution to the signal is therefore an unphysical matrix element that does not conserve energy. We avoid this difficulty without resorting to multi-exponential fits to the time dependence by using antiperiodic spatial boundary conditions on the down quark propagator for $A_{2}$ and G-parity spatial boundary conditions for $A_{0}[6,7,8,9]$ : Both have the effect of imposing antiperiodic boundary conditions on the pions, thus increasing their ground-state momentum from 0 to $\pi / L$, where $L$ is the lattice box size. By appropriately tuning $L$ we can therefore match the kaon and $\pi \pi$ energies.

\subsection{Results and outlook}

We have computed $A_{2}$ [10] on our $48^{3} \times 96$ and $64^{3} \times 1282+1$ flavor Möbius domain wall fermion (DWF) ensembles with the Iwasaki gauge action, which have large, $5.4 \mathrm{fm}$ physical volumes ensuring small finite-volume errors, and lattice spacings of $a^{-1}=1.73 \mathrm{GeV}$ and $2.36 \mathrm{GeV}$, respectively, allowing us to take the continuum limit. The final results for $\operatorname{Re}\left(A_{2}\right)$ and $\operatorname{Im}\left(A_{2}\right)$ have $3 \%$ statistical errors and $10 \%$ and $12 \%$ total errors, respectively, which are dominated by the errors due to the truncation of the perturbative series used to compute the Wilson coefficients and in the perturbative matching of our RI-SMOM renormalized lattice operators to $\overline{\mathrm{MS}}$.

The calculation of $A_{0}$ [1] has thus far been performed only with a single, somewhat coarse lattice spacing of $a^{-1}=1.38 \mathrm{GeV}$ but again with a large physical volume of $4.5 \mathrm{fm}$. We utilize a $32^{3} \times 64$ volume with $2+1$ flavor Möbius DWF and the Iwasaki+DSDR gauge action. We obtain $21 \%$ and $65 \%$ statistical errors on $\operatorname{Re}\left(A_{0}\right)$ and $\operatorname{Im}\left(A_{0}\right)$, respectively; the latter being enhanced by a $50 \%$ numerical cancellation of the dominant $Q_{4}$ and $Q_{6}$ contributions. The systematic error is again dominated by the $15 \%$ error arising from the truncation of the perturbative series in computing the Wilson coefficients and $\overline{\mathrm{MS}}$ matching factors, here exacerbated by the somewhat low $1.53 \mathrm{GeV}$ renormalization scale demanded by the coarse lattice spacing.

Combining the lattice values of $\operatorname{Im}\left(A_{2}\right)$ and $\operatorname{Im}\left(A_{0}\right)$ and the $\pi \pi$-scattering phase shifts computed alongside, with the experimental values for $\operatorname{Re}\left(A_{2}\right)$ and $\operatorname{Re}\left(A_{0}\right)$ (While our lattice values agree with experiment to within statistics, the latter are more precisely determined and are not expected to be significantly impacted by BSM physics.) we obtain the following result

$$
\operatorname{Re}\left(\frac{\varepsilon^{\prime}}{\varepsilon}\right)=1.38(5.15)(4.59) \times 10^{-4}
$$


where the errors are statistical and systematic, respectively. Our result has roughly $3 \times$ the experimental error and agrees with experiment to $2.1 \sigma$. However there is a hint of a tension that has provoked much interest both within and outside the lattice community, providing strong motivation for continued study.

These calculations also allow us to examine a long-standing puzzle, namely the origin of the $\Delta I=1 / 2$ rule - a factor of 22.5 enhancement of $\operatorname{Re}\left(A_{0}\right)$ over $\operatorname{Re}\left(A_{2}\right)$. While a factor of 2 is obtained through the running of the Wilson coefficients to the charm scale, it remained an open question as to whether the remaining factor of 10 originates from new physics or due to non-perturbative QCD effects. Combining our lattice values we obtain

$$
\frac{\operatorname{Re}\left(A_{0}\right)}{\operatorname{Re}\left(A_{2}\right)}=31.1(11.2)
$$

which, while having large errors, is consistent with the experimental value suggesting non-perturbative QCD gives rise to the enhancement. We can trace the origin of this large ratio to a strong, highly quark-mass dependent, 70\%-level numerical cancellation between the two dominant contractions in the $I=2$ channel [10] that serves to heavily suppress $\operatorname{Re}\left(A_{2}\right)$.

Our main priority for the near future is to increase statistics on the $A_{0}$ calculation, and to this end we have started a large-scale measurement programme with the target of a $4 \times$ increase in the number of measurements within the year. We have also started work on reducing the perturbation theory truncation systematic by raising the renormalization scale from $1.53 \mathrm{GeV}$ to $2.29 \mathrm{GeV}$ using the step-scaling procedure; preliminary results [11] suggest a factor of 2 reduction in the systematic error can be achieved. On a longer timescale we intend to generate a second lattice spacing in order to take the continuum limit of $A_{0}$. We also aim to investigate the effects of electromagnetism and isospin-breaking effects as well as to treat the charm quark non-perturbatively.

\section{3. $K_{L}-K_{S}$ mass difference}

Neglecting small CPV effects, the mixing of neutral kaons induced by second-order weak interactions gives rise to a mass difference between the CP-eigenstates $K_{1} \sim K_{L}$ and $K_{2} \sim K_{S}$ :

$$
\Delta M_{K}=2 \sum_{n} \frac{\left\langle\bar{K}^{0}\left|H_{W}^{\Delta S=1}\right| n\right\rangle\left\langle n\left|H_{W}^{\Delta S=1}\right| K^{0}\right\rangle}{M_{K}-E_{n}}=3.483(6) \times 10^{-12} \mathrm{MeV},
$$

This quantity is extremely small because it arises due to flavour-changing neutral currents (FCNC) and is therefore highly suppressed in the Standard Model by the GIM mechanism. This makes is an attractive quantity to examine for hints of BSM FCNC.

$\Delta M_{K}$ can be computed using using the $\Delta S=2$ weak effective theory, with the charm quark is integrated out perturbatively. For this quantity perturbation theory is poorly convergent at the charm scale, giving rise to a $36 \%$ systematic error on the phenomenological result. These calculations also neglect non-perturbative long-distance effects arising when the two weak Hamiltonian operators are separated by distances $\sim 1 / \Lambda_{\mathrm{QCD}}$.

We can use lattice techniques to evaluate the matrix element in Eq. 3.1 directly. This contains two insertions of the $\Delta S=1$ weak effective Hamiltonian, between which propagate all intermediate states comprising light and charm quarks. In order to avoid using perturbation theory at the charm scale it is also necessary to directly include a charm quark in the lattice calculation.

The matrix element of the two weak effective Hamiltonian operators between $K$ and $\bar{K}$ states shown in Figure 1 evaluates to the following: 


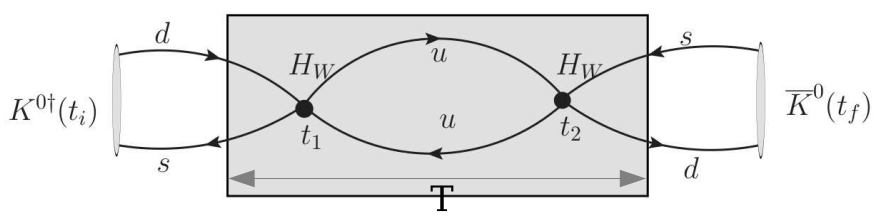

Figure 1: Layout of the $\Delta M_{K}$ calculation showing the source and sink kaon operators and the two weak Hamiltonian insertions. The integration region in which we vary the temporal locations of the weak Hamiltonian operators is indicated by the gray box.

$$
\mathscr{A}=N_{K}^{2} e^{-M_{K}\left(t_{i}-t_{f}\right)} \sum_{n} \frac{\left\langle\bar{K}^{0}\left|H_{W}^{\Delta S}\right| n\right\rangle\left\langle n\left|H_{W}^{\Delta S=1}\right| K^{0}\right\rangle}{M_{K}-E_{n}}\left(-T-\frac{1}{M_{K}-E_{n}}+\frac{e^{\left(M_{K}-E_{n}\right) T}}{M_{K}-E_{n}}\right)
$$

where $N_{K}$ is the amplitude of the kaon source operator acting on the vacuum. The desired quantity can be extracted by fitting the term linear in integration interval $T$.

The key challenges of this calculation are as follows:

- For physical kinematics, two- and three-pion intermediate states propagate between the two operator insertions, and, as with the $K \rightarrow \pi \pi$ calculation, these require Lellouch-Lüscher corrections for finite-volume errors.

- For intermediate states of energy less than the kaon mass the final term in Eq 3.2 gives rise to an unphysical exponentially growing background that must be explicitly subtracted.

- Large statistics are required again due to the presence of disconnected diagrams.

- A valence charm quark is required to avoid the use of perturbation theory and also to enable the GIM mechanism to remove a divergence occuring when the operators approach each other. This requires a fine lattice spacing in order to minimize discretization errors.

\subsection{Results and outlook}

We have computed $\Delta M_{K}$ on a $24^{3} \times 64$ Shamir DWF ensemble with the Iwasaki gauge action and a lattice spacing of $a^{-1}=1.73 \mathrm{GeV}$ [12]. In order to avoid having to correct multi-particle intermediate states for the finite-volume, we used unphysically heavy, $330 \mathrm{MeV}$ pions such that only the single-pion intermediate state is lighter than the kaon. We also used an unphysically light charm quark mass of $949 \mathrm{MeV}$ so as to avoid large discretization errors. The calculation was performed with very high statistics - 800 configurations - and the matrix element was maximally translated across the lattice for optimal use of each configuration.

We obtain the following result:

$$
\Delta M_{K}=3.19(41)(96) \times 10^{-12} \mathrm{MeV}
$$

where the parentheses give the statistical error and the discretization systematic, respectively. This result is consistent with the experimental value despite the unphysical masses. An interesting observation is that the disconnected diagrams have a large contribution, which is in contradiction with the "OZI rule", which states that disconnected diagrams where the initial and final states are connected only by internal gluon lines are suppressed because the moderate-energy gluons required to carry the energy have reduced coupling due to the running of the coupling constant.

The dominant error on our result for unphysical kinematics arises due to the proximity of the charm quark mass to the bound associated with the inverse lattice spacing, which gives rise to an 
estimated $30 \%$ discretization error. In the future we aim to use a finer lattice for this calculation, as well as to move to physical pion masses.

\section{Rare kaon decays}

Lattice simulations can be used to study additional FCNC processes in the Standard Model. Among these, of particular interest are the rare kaon decays $K^{+} \rightarrow \pi^{+} l^{+} l^{-}$and $K_{s} \rightarrow \pi^{0} l^{+} l^{-}$. These amplitudes are dominated by long-distance contributions and therefore cannot be reliably computed using perturbation theory. On the lattice we compute the QCD part of the diagram, $K \rightarrow \pi \gamma^{*}$, and use a moving pion to study the photon energy dependence. The techniques required are similar to those employed in the calculation of $\Delta M_{K}$ above, only with replacing one of the effective Hamiltonian insertions with an electromagnetic current insertion.

We are currently performing an exploratory study of these matrix elements using the $24^{3} \times 64$ ensembled mentioned above, using unphysically large pion and kaon masses of $430 \mathrm{MeV}$ and 625 $\mathrm{MeV}$, respectively, and an unphysically light charm quark mass of $533 \mathrm{MeV}$. Preliminary results using only the connected diagrams can be found in Refs. $[13,14,15]$. While we have not obtained a signal for the amplitude due to the absence of disconnected diagrams, we have demonstrated the efficacy of our approach and have laid the groundwork for extending to physical masses. The largest barrier is moving to a physical charm mass, which may be avoided if we work in the threeflavor theory, as in the $K \rightarrow \pi \pi$ calculation, where the charm has been integrated out perturbatively. However phenomenological results suggest that the charm contribution to these decays may be large and therefore it may be necessary to simulate the charm directly.

FCNC also enter in the rare kaon decays $K \rightarrow \pi v \bar{v}$. Unlike the above these are short-distance dominated and the long-distance component is expected to be only $\mathscr{O}(5 \%)$; however the NA62 experiment is expected to measure these decays to $10 \%$ precision, such that precise knowledge of the long-distance component will soon be necessary. These processes require calculating a larger set of operators that includes an effective vertex representing the Z-boson coupling, and shortdistance divergences must be handled by matching the bilocal operator to a point-like four-quark operator. An exploratory calculation of this matrix element has been performed on a $16^{3} \times 32$ ensemble with $a^{-1}=1.73 \mathrm{GeV}$ and unphysical masses similar to those used above [16], again demonstrating that this calculation is possible with current lattice techniques.

\section{References}

[1] Z. Bai et al. Phys. Rev. Lett. 115, no. 21, 212001 (2015) [arXiv:1505.07863 [hep-lat]].

[2] L. Lellouch and M. Luscher, Commun. Math. Phys. 219, 31 (2001) [hep-lat/0003023].

[3] G. Martinelli et al., Nucl. Phys. B 445, 81 (1995) [hep-lat/9411010].

[4] C. Sturm et al., Phys. Rev. D 80, 014501 (2009) [arXiv:0901.2599 [hep-ph]].

[5] J. Foley et al.,Comput. Phys. Commun. 172, 145 (2005) [hep-lat/0505023].

[6] C. h. Kim and N. H. Christ, Nucl. Phys. Proc. Suppl. 119, 365 (2003) [hep-lat/0210003].

[7] U. J. Wiese, Nucl. Phys. B 375, 45 (1992).

[8] C. Kim, Nucl. Phys. Proc. Suppl. 129, 197 (2004) [hep-lat/0311003].

[9] C. Kelly PoS LATTICE 2012, 130 (2012).

[10] T. Blum et al., Phys. Rev. D 91, no. 7, 074502 (2015) [arXiv:1502.00263 [hep-lat]].

[11] C. Kelly, PoS LATTICE 2016, 308 (2016)

[12] Z. Bai et al., Phys. Rev. Lett. 113, 112003 (2014) [arXiv:1406.0916 [hep-lat]].

[13] N. H. Christ et al., Phys. Rev. D 92, no. 9, 094512 (2015) [arXiv:1507.03094 [hep-lat]].

[14] N. Christ et al., PoS LATTICE 2015, 340 (2016) [arXiv:1602.01374 [hep-lat]].

[15] N. H. Christ et al., arXiv:1608.07585 [hep-lat].

[16] N. H. Christ et al., PoS CD 15, 033 (2016). 